\title{
Antibiotic Susceptibility Pattern of Pseudomonas aeruginosa Isolates from Wound Infections in a Tertiary Care Centre in South Kerala, India
}

\author{
Suresh Babu \\ Department of Microbiology, Dr SM CSI Medical College and Hospital, Trivandrum, India \\ Email address: \\ sureshbabu6831@yahoo.in

\section{To cite this article:} \\ Suresh Babu. Antibiotic Susceptibility Pattern of Pseudomonas aeruginosa Isolates from Wound Infections in a Tertiary Care Centre in \\ South Kerala, India. International Journal of Infectious Diseases and Therapy. Vol. 3, No. 2, 2018, pp. 30-33. \\ doi: $10.11648 /$ j.ijidt.20180302.12
}

Received: May 4, 2018; Accepted: June 14, 2018; Published: July 9, 2018

\begin{abstract}
Multiple antibiotic resistance in bacterial populations is a pervasive and growing clinical problem, which is recognized as a threat to public health. Drug resistance to Pseudomonas sp. has spread to such a level irrespective of the type of patients, that, its pattern of distribution and antibiotic resistance needs to be studied in detail, especially in trauma patients. Of the Gram negative bacilli, Pseudomonas aeruginosa has been of particular interest, the incidence of which in wound infection has increased compared to a decade back. The objective of this study was to know the antibiotic susceptibility pattern of Pseudomonas aeruginosa isolates from wound infections. Out of the total of 1404 cases from which pus samples collected, 204 (14.5\%) yielded Pseudomonas aeruginosa isolates. Among the antibiotics tested, Imipenem was the most sensitive drug, showing susceptibility in $81.3 \%$ of the isolates, followed by Piperacillin + tazobactam (76.4\%), Meropenem (70.5\%) and Piperacillin (67.6\%). Fifteen (7.4\%) multidrug resistant strains were reported out of the 204 isolations. Increase in resistance to aminoglycosides and fluoroquinolones in this study, prompted evaluation of antibiotic susceptibility pattern of Pseudomonas isolates from clinical samples at regular intervals.
\end{abstract}

Keywords: Pseudomonas aeruginosa, Multiple Drug Resistance, Wound Infections, Gram Negative Bacilli

\section{Introduction}

Wound infection is universal and the bacterial type varies with geographical location, resident flora of the skin and clothing at the site of wound [1]. There are reports of considerable number of carriers for $P$. aeruginosaamong the healthy people within the hospital environment, capable of transmitting infection to the patients undergoing treatment [2]. In the recent years, Pseudomonas aeruginosa has overtaken Staphylococcus aureus in causing wound infections [3]. In the past three years, some of the Indian studies shown Pseudomonas sp as the most predominant isolate from wound infections (Gunjam Shrivastava et al, 2016 [4], Vivek Kulkami et al, 2015 [5] and Richa Gupta et al, 2016 [6]. In one of the studies from Maharashtra, Sapana G Mundhada et al, 2015 [7] reported Pseudomonas aeruginosa as second dominant strain isolated, wherein Klebsiella sp was the most predominant isolation. In yet another study from Odisha, Pseudomonas trailed behind
Staphylococcus aureus and Klebsiella, among the isolated bacterial pathogens from wound infections (Santa Otta et al, 2015 [8]. Suryakala R Nair et al, 2015 [9], showed that Pseudomonas aeruginosa was the second predominant isolation behind Staphylococcus aureus, from diabetic foot ulcers. The work was carried out from Trivandrum, Kerala. This organism demonstrates resistance to multiple antibiotics, there by jeopardizing the selection of appropriate treatment [10]. Carbapenem resistance is the dominant one due to loss of carbapenem specific porin OprD2 [11]. There are few studies from India that provide antimicrobial susceptibility of $P$. aeruginosa isolates $[12,13]$. But, there are, so far, no reports of this nature from this part (Karakonam, Trivandrum district) of South Kerala, India.

\section{Materials and Methods}

This work was carried out in the department of Microbiology, Dr. SM CSI Medical College, Karakonam, 
Trivandrum district, Kerala, India. Pus and discharges from various wound infections, mostly from inpatients, were collected for a 11 months period from January 2017 to November 2017. The samples collected were from surgery, ENT, medicine, intensive care units, critical care units, OG, dermatology, peripheral centre and OPDs. Out of the total of 1404 samples collected, 204 (14.5\%) yielded Pseudomonas aeruginosa on culture. The media used were blood agar and MacConkey agar from HiMedia Mumbai. The isolations were identified based on colony and biochemical characteristics and also bluish- green diffusible pigment production (Figure 1).

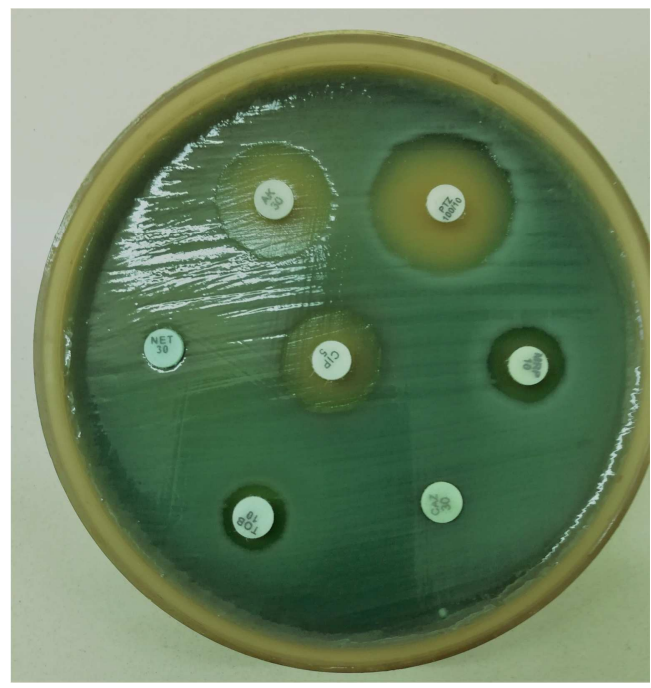

Figure 1. Pigment production and Antibiotic susceptibility pattern of Pseudomonas aeruginosa isolate- Kirby Bauer disc diffusion method.

Pseudomonas aeruginosa strains were subjected to antibiotic susceptibility testing by Kirby- Bauer disc diffusion method in Mueller Hinton agar, HiMedia, Mumbai, (Figure 1), based on the CLSI guide lines [14, 15]. ATCC strains of Pseudomonas aeruginosa (ATCC 27853) and Escherichia coli (ATCC 25922) were used as quality control. All isolates in this study were non-duplicate. The antibiotic discs used were Amikacin (30 mcg), Gentamicin (20 mcg), Piperacillin (100 mcg), Piperacillin + Tazobactam $(10 \mathrm{mcg})$, Imipenem $(10 \mathrm{mcg})$, Cefoperazone $(75 \mathrm{mcg})$, Ceftazidime (30 mcg), Netilmicin (30 mcg), Ciprofloxacin (5 mcg) and Meropenem (10 mcg), purchased from HiMedia, Mumbai. After performing the test, the plates were incubated at $37^{\circ} \mathrm{C}$ overnight. The diameter of the zone of inhibition was measured and compared to that of standard strains and the results were interpreted as sensitive or resistant, based on CLSI guidelines [16].

\section{Results}

Out of the total of 1404 samples cultured, 204 (14.5\%) yielded Pseudomonas aeruginosa. Male patients predominated over the females in the number Pseudomonas isolates obtained (Table 1).

Table 1. Sex wise distribution of Pseudomonas isolates $(n=204)$.

\begin{tabular}{lll}
\hline Sex & Male & Female \\
\hline No. of isolates & 130 & 74 \\
$\%$ of isolates & 63.7 & 36.3 \\
\hline
\end{tabular}

The maximum number of Pseudomonas isolations were made from the age group of $>60(33.8 \%)$, followed by $51-60$ and 41-50 (Table 2).

Table 2. Age wise distribution of Psuedomonasisolates $(n=204)$.

\begin{tabular}{llllllll}
\hline $\begin{array}{l}\text { Age } \\
\text { group }\end{array}$ & $\mathbf{0 - 1 0}$ & $\mathbf{1 1 - 2 0}$ & $\mathbf{2 1 - 3 0}$ & $\mathbf{3 1 - 4 0}$ & $\mathbf{4 1 - 5 0}$ & $\mathbf{5 1 - 6 0}$ & $>\mathbf{6 0}$ \\
\hline $\begin{array}{l}\text { No. of } \\
\text { isolates } \\
\% \text { of } \\
\text { isolates }\end{array}$ & 9 & 2 & 11 & 14 & 35 & 64 & 69 \\
\hline
\end{tabular}

The most number of isolations were made from the samples received from male surgical ward (MSW) $\{31.9 \%\}$, followed by a satellite multi- speciality hospital attached to this medical college, with equal distributions from female surgical ward and ENT department, in that order (Table 3).

Table 3. Ward wise distribution of Pseudomonas isolates ( $n=204)$.

\begin{tabular}{|c|c|c|c|c|c|c|c|c|c|c|c|}
\hline Ward & MSW & FSW & ENT ward & KK & MMW & MICU & SICU & $\mathrm{CCU}$ & ANW & Skin ward & OP \\
\hline $\begin{array}{l}\text { No. of } \\
\text { isolates }\end{array}$ & 65 & 23 & 23 & 40 & 7 & 4 & 4 & 1 & 1 & 1 & 35 \\
\hline $\begin{array}{l}\% \text { of } \\
\text { isolates }\end{array}$ & 31.9 & 11.2 & 11.2 & 19.6 & 3.4 & 2 & 2 & 0.5 & 0.5 & 0.5 & 17.2 \\
\hline
\end{tabular}

MSW- Male surgical ward, FSW- Female surgical ward, KK- peripheral multi-speciality hospital, MMW- Male medical ward

Among the antibiotics used, the maximum number of Pseudomonas isolates were found sensitive to Imipenem (81.3\%), followed by Piperacillin + tazobactam (76.4\%), Meropenem (70.5\%) and Piperacillin (67.6\%), (Table 4, 5) and (Figure 2).

Table 4. Antibiotic sensitivity pattern of Pseudomonas isolates $(n=204)$.

\begin{tabular}{|c|c|c|c|c|c|c|c|c|c|c|c|c|}
\hline Antibiotics & Ak & & G & & Pc & & $\mathbf{P t}$ & & $\mathbf{T b}$ & & Im & \\
\hline & $\mathbf{S}$ & $\mathbf{R}$ & $\mathbf{S}$ & $\mathbf{R}$ & $\mathbf{S}$ & $\mathbf{R}$ & $\mathbf{S}$ & $\mathbf{R}$ & $\mathbf{S}$ & $\mathbf{R}$ & $\mathbf{S}$ & $\mathbf{R}$ \\
\hline No. of isolates & 132 & 72 & 100 & 104 & 138 & 66 & 156 & 48 & 102 & 102 & 166 & 38 \\
\hline$\%$ of isolates & 64.7 & 35.3 & 49 & 51 & 67.6 & 32.4 & 76.4 & 23.6 & 50 & 50 & 81.3 & 18.7 \\
\hline
\end{tabular}

Ak- Amikacin, G- Gentamicin, Pc- Piperacillin, Pt- Piperacillin+ tazobactum, Tb- Tobramycin, Im- Imipenem 
Table 5. Antibiotic sensitivity pattern of Pseudomonas isolates $(n=204)$.

\begin{tabular}{lllllllll}
\hline Antibiotics & Cip & & Mrp & & Cpz & & Caz & Nt \\
\hline & S & R & S & R & S & R & S & R \\
\hline No. of isolates & 101 & 103 & 144 & 60 & 124 & 80 & 134 & 70 \\
\% of isolates & 49.5 & 50.5 & 70.5 & 29.5 & 60.7 & 39.3 & 65.6 & 34.4 \\
\hline
\end{tabular}

Cip- Ciprofloxacin, Mrp- Meropenem, Cpz- Cefoperazone, Caz- Ceftazidime, Nt- Netilmicin

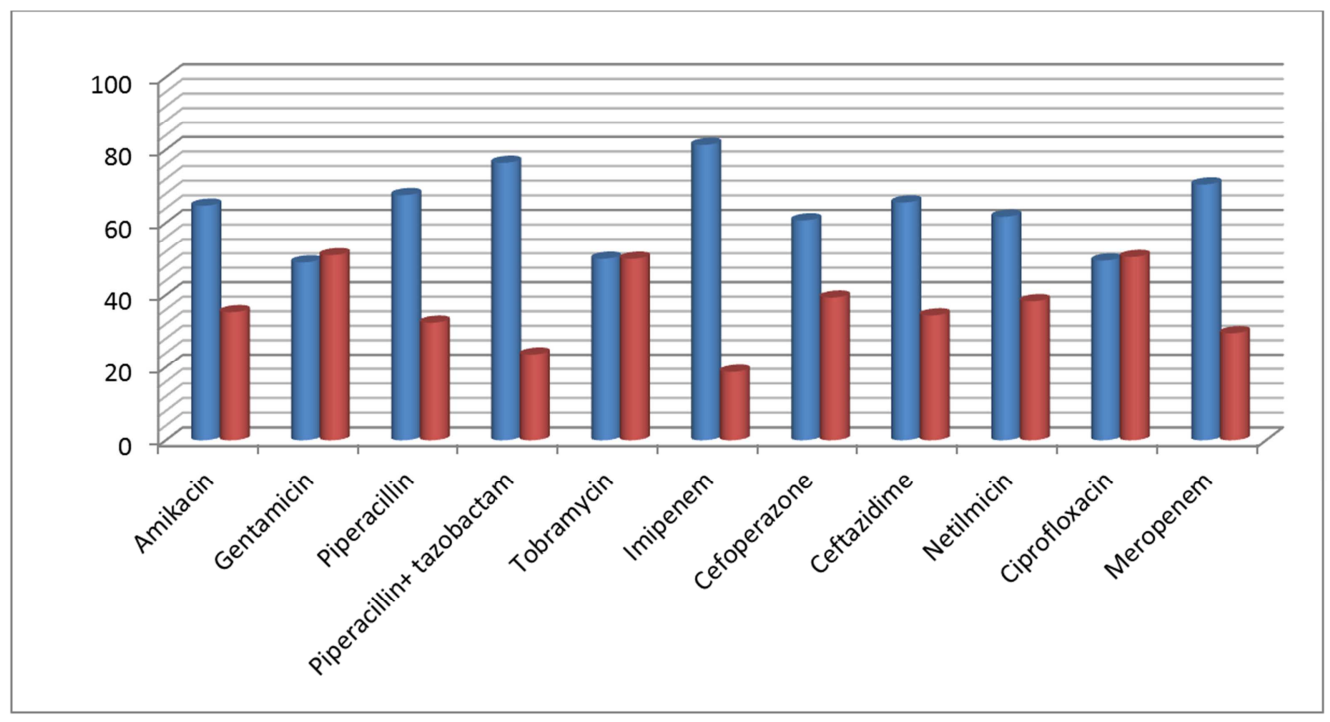

Figure 2. Antibiotic susceptibility pattern of Pseudomonas isolates in $\%(n=204)$.

Among the $3^{\text {rd }}$ generation cephalosporins used, Ceftazidime and Cefoperazone recorded sensitivity of $65.6 \%$ and $60.7 \%$ respectively. Of the aminoglycosides, Amikacin, Netilmicin and Gentamicin recorded $64.7 \%, 61.7 \%$ and $49 \%$ respectively. Ciprofloxacin amounted $49.5 \%$ and Tobramycin showed $50 \%$ sensitivity. Fifteen $(7.4 \%)$ multidrug resistant strains were reported out of the total 204 isolations.

\section{Discussion}

The isolation rate of Pseudomonas aeruginosa of $14.5 \%$ in our study was less than that of another study of wound infections in Varanasi, India (32\%), [17]. The age group of $>60$ and 51-60 accounting for maximum number of isolations, in our study, more or less correlates with another study in North India [17]. Predominant number of Pseudomonas isolates made from cases in male surgical ward in this study differ from another report from New Delhi, India, wherein, neurosurgery cases dominated [18]. Most number of isolates being sensitive to Imipenem $(81.3 \%)$, Piperacillin+ Tazobactum (76.4\%), and Piperacillin (67.6\%), in the decreasing order, in our study is in correlation with Deepak Juyal et al [19] and Viren A Javia et al [20], wherein, they showed a sensitivity pattern of $71.3 \%, 69.2 \%$ and $52 \%$ respectively. A novel finding in this study was that Imipenem, Piperacillin + Tazobactam, andPiperacillin were found to be sensitive to more number of isolates, than as reported by the studies quoted above. Among the aminoglycosides, Amikacin and Gentamicin recorded 64.7\% and $49 \%$ sensitivity, respectively in our study, which is more or less in correlation with Deepak Juyal et al, wherein they reported $72 \%$ and $48.9 \%$ correspondingly [19]. Although, Ciprofloxacin in our study showing a sensitivity of $49.5 \%$, is well above the $24.5 \%$ as mentioned in the same study as quoted above [19]. Anyway, as per our findings, there is a gradual and constant increase in resistance to aminoglycosides and fluoroquinolones, which is a matter of concern. 15 MDR strains also reported in this study added up to the agony, and as a result, evaluation of susceptibility pattern of Pseudomonas aeruginosa strains isolated at regular intervals is required for effective treatment for those cases.

\section{Conclusion}

In our study on antibiotic susceptibility pattern of Pseudomonas aeruginosa isolates, the percentage of resistant strains was 51 and 35.3 respectively against Gentamicin and Amikacin. $34.4 \%$ were resistant to Ceftazidime, a $3^{\text {rd }}$ generation cephalosporin. $50.5 \%$ of the strains were resistant to Ciprofloxacin. Piperacillin showed $32.4 \%$ resistance. So, infections due to those Pseudomonas strains have to be treated only after performing the antibiotic susceptibility testing by including these drugs. 15 multidrug resistant strains emerging out of the 204 total isolates, prompts one to detect the gene responsible for such resistance in future studies. In our study, the most number of strains were susceptible to Imipenem $(81.3 \%)$, Piperacillin + tazobactum (76.4\%) and Piperacillin (67.6\%), which could be used as reserve drugs in case of severe illnesses, due to Pseudomonas infection. Increase in resistance to antibiotics used other than Imipenem and Piperacillin + tazobactam and also emergence of 15 MDR strains of Pseudomonas aeruginosa in our study 
is a matter of concern in all health care establishments. As a result regular antibiotic susceptibility testing of Pseudomonas isolates is required for effective treatment of those cases.

\section{Acknowledgements}

I am thankful to Dr. S. Rema Devi, Professor and Head of the Department of Microbiology, Dr SM CSI Medical College and Hospital, Karakonam, Kerala, India, for permitting me, as well as extending work related expertise to complete this work. I am also grateful to the Director and the Principal of this Institution for the constant encouragement in research related activities.

\section{References}

[1] Oguntibeju OO, Nwobu RA. Occurrence of P. aeruginosa in post operative wound infection. Pak J Med Sc 2004; 20:18791.

[2] Kolmos HJ, Svendsen RN, Nielsen SV. The surgical team as a resource of post - operative wound infections. J Hosp Infect 1997; 35:207-14.

[3] Joshi KR, Onaghise EO, Oyaide SM. Aeruginosine typing of $P$. aeruginosa isolated at the university of Benin teaching Hospital, Benin. Afr J Clin Microbiol 1984; 1:13-8.

[4] Ganjam Shrivastava, GS Bhatambare, A Lunavat, KB Patel, T Maheshwari. Pattern of microorganisms from burn wounds and their trends of susceptibility to antibiotics in tertiary care centre. Int J of Health System and Disaster Management 2016; $4(2): 53-57$.

[5] Vivek Kulkami, Sagar Mohan Arati, YM Jayaraj, Channappa T Shivannavar, MR Joshi. Bacterial etiology and their biogram in burn wound infections at Kalaburgi region (India). Ind J Burns 2015; 23(1):65-70.

[6] Richa Gupta, Abida Malik, Meher Rizvi, Moied Ahmed. Presence of metallo beta lactamases (MBL), ESBL Amp C positive non- fermenting gram negative bacilli among intensive care unit patients with special reference to molecular detection of bla CTX-M and bla Amp C genes. Ind J Med Res. 2016; 144(2):271-275

[7] Sapana G Mundhada, Prakash H Waghmare, Prachala G Rathod, Kishore V Ingole. Bacterial and fungal profile of burn wound infections in tertiary care centre. Ind J Burns. 2015; 23(1):71-75.

[8] Santa Otta, Jayant Kumar Dash, Bichitrananda Swain. Aerobic bacteriology of burn wound infections. CHRISMED J Health Res 2015; 2(4):337-341.
[9] Suryakala R Nair, Resmi Rajan, Sharada Devi Karunakaran, Lalithabai. A clinicomicrobiological study of diabetic foot ulcers from South Kerala. J of the Academy of Clinical Microbiologists. 2015; 17(2):94-99.

[10] Obritsch MD, Fish DN, McLaren R, Jung R. National Surveillance of Antimicrobial Resistance in Pseudomonas aeruginosa isolates obtained from Intensive Care Unit Patients from 1993 to 2002. Antimicrob Agents Chem 2004; 48:460610 .

[11] Quinn JP, Studemeister AE, DiVincenzo CA, Lerner SA. Resistance to imipenem in Pseudomonas aeruginosa: Clinical experience and biochemical mechanisms. Rev Infect Dis $1988 ; 10: 892-8$.

[12] Malini A, Deepa EK, Gokul BN, Prasad SR. Nonfermenting gram-negative bacilli infections in a tertiary care hospital in Kolar, Karnataka. J Lab Physicians 2009; 1:62-6.

[13] Taneja N, Maharwal S, Sharma M. Imipenem resistance in nonfermenters causing nosocomial urinary tract infections. Indian J Med Sci 2003; 57:294-9.

[14] Clinical and Laboratory Standards Institute. Performance Standards for Antimicrobial Susceptibility testing; Twentieth Informational Supplement (June 2010 update). M100-S20. Pennsylvania, USA: Clinical and Laboratory Standard Institute (CLSI) Document; 2010.

[15] Clinical and Laboratory Standards Institute. Performance Standards for Antimicrobial Susceptibility testing; TwentySecond Informational Supplement. M100-S22. Wayne, Pennsylvania, USA: Clinical and Laboratory Standards Institute (CLSI) Document; 2012.

[16] National Committee for Clinical Laboratory Standards. Performance standards for antimicrobial disk susceptibility tests. Approved standard M2 A7 NCCLS, Villanova, PA: 1995. p. 15.

[17] ShampaAnupurba, AmitabhaBattacharjee, et al. Antimicrobial susceptibility of Pseudomonas aeruginosaisolated from wound infections. Indian J Dermatology 2006; 51(4):286-88.

[18] NonikaRajkumari, NibuVargheese John, PurvaMathur, et al. Antimicrobial resistance in Pseudomonas $s p$. causing infections in trauma patients. A six year experience from a South Asian Country. J of Global Inf Dis 2014; 6(4):182-85.

[19] Deepak Juyal, RajatPrakash, Shamanth A Shanakarnarayan, et al. Prevalence of non- fermenting gram negative bacilli and their vitro susceptibility in a tertiary care hospital of Uttarkhand: A study from foothills of Himalayas. Saudi J Health Sci 2013; 2(2):108-112.

[20] Viren A Javiya, Samsuvra B Ghatak, Kamlesh R Patel et al. Antibiotic susceptibility patterns of Pseudomonas aeruginosa at a tertiary care hospital in Gujarat, India. Ind J Pharmacology 2008; 40(5):230-234. 